\title{
Monopole Condensation in Lattice SU(2) QCD *
}

\author{
Tsuneo Suzuki ${ }^{\dagger}$ \\ Department of Physics, Kanazawa University, Kanazawa 920-11, Japan
}

\begin{abstract}
This is the short review of Monte-Carlo studies of quark confinement in lattice QCD. After abelian projections both in the maximally abelian and Polyakov gauges, it is seen that the monopole part alone is responsible for confinement. A block spin transformation on the dual lattice suggests that lattice $S U(2)$ QCD is always ( for all $\beta$ ) in the monopole condensed phase and so in the confinement phase in the infinite volume limit.
\end{abstract}

\section{Introduction}

It is crucial to understand the mechanism of quark confinement in order to explain hadron physics out of QCD. Our standpoint is based on the 'tHooft idea of abelian projection of QCD. 1 The abelian projection is to fix the gauge in such a way that the maximal torus group remains unbroken. After the abelian projection, monopoles appear as a topological quantity in the residual abelian channel. QCD is reduced to an abelian theory with electric charges and monopoles. If the monopoles make Bose condensation, charged quarks and gluons are confined due to the dual Meissner effect.

Based on this standpoint, the present author and his collaborators have studied color confinement mechanism and hadron physics performing Monte Carlo simulations of abelian projection in lattice QCD. 15 The aim of the study is to ascetain correctness of the picture, that is, to check if monopole condensation really occurs in QCD. Here I review the results of these studies compactly. ${ }^{\text {. }}$

\section{Abelian dominance and monopole dominance}

\subsection{Maximally abelian and Polyakov gauges}

There are infinite ways of abelian projection extracting such an abelian theory out of QCD. We have found two gauges which show interesting behaviors called abelian dominance.212 21

${ }^{*}$ This is based on works done in collaboration with Y.Matsubara, H.Shiba, S.Kitahara, S.Ejiri, S.Okude and K.Yotsuji.

${ }^{\dagger}$ E-mail address: suzuki@hep.s.kanazawa-u.ac.jp

* We have also studied hadron physics based on an infrared effective Lagrangian constructed directly from QCD on the assumption of the above picture, 160 but this time I skip all of them. 
SU(2) MA GAUGE

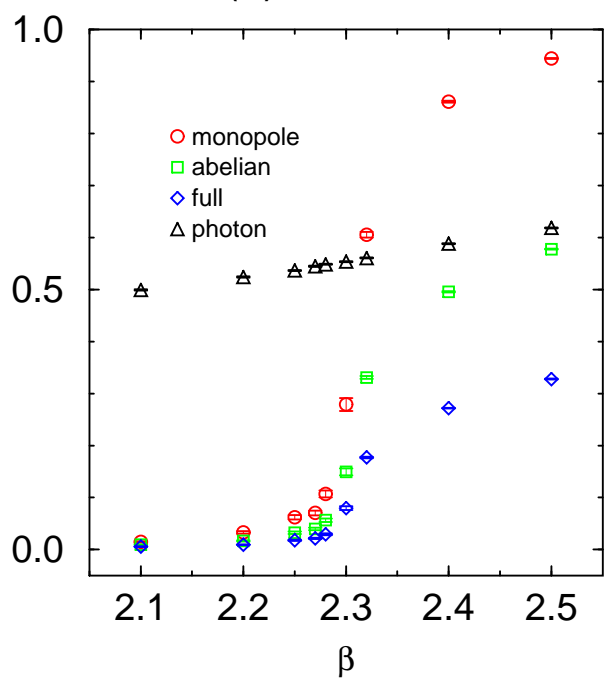

Fig.1 Monopole Dirac string and photon contributions to Polyakov loops in MA gauge.
SU(2) POLYAKOV GAUGE

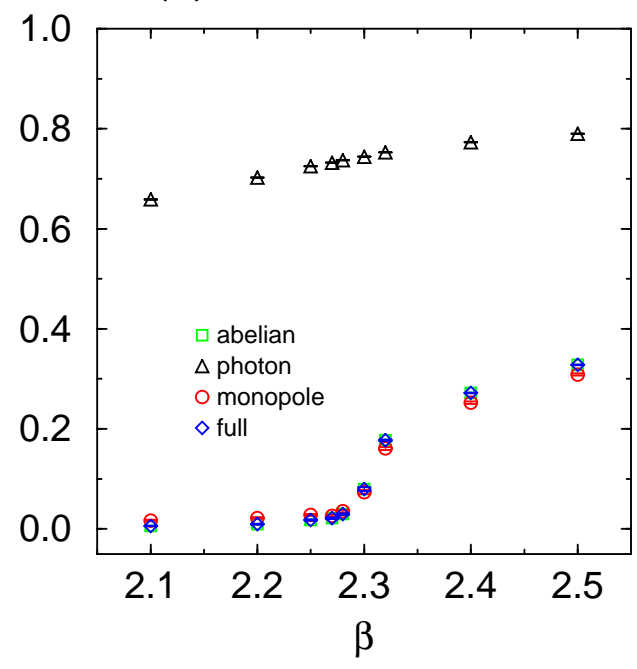

Fig.2 Monopole Dirac string and photon contributions to Polyakov loops in the Polyakov gauge.

One is the maximally abelian (MA) gauge. Define a matrix in $S U(2)$ QCD

$$
\begin{aligned}
X(s) & =\sum_{\mu}\left[U(s, \mu) \sigma_{3} U^{\dagger}(s, \mu)+U^{\dagger}(s-\hat{\mu}, \mu) \sigma_{3} U(s-\hat{\mu}, \mu)\right] \\
& =X_{1}(s) \sigma_{1}+X_{2}(s) \sigma_{2}+X_{3}(s) \sigma_{3} .
\end{aligned}
$$

Then a gauge satisfying $X_{1}(s)=X_{2}(s)=0$ is the MA gauge21 which tends to a $U(1)$ covariant gauge $\left(\partial_{\mu} \pm i g A_{\mu}^{3}\right) A^{ \pm \mu}=0$ in the continuum limit. The other is the Polyakov gauge which is defined by diagonalizing Polyakov loop operators. We have found that important features of confinement, i.e., the string tension and the charateristic behaviors of the Polyakov loops are well reproduced in terms of the abelian operators $O(u(s, \mu))$ both in MA gauge胭 and in the Polyakov gauge. 1 See Figures $1 \sim 4$.

\subsection{Monopole dominance in the Polyakov loops}

An abelian Polyakov loop $P$ which is written in terms of abelian link fields alone is given by a product of contributions from Dirac strings of monopoles and from photons 10.12 We have observed the photon and the Dirac-string contributions separately. See Figs.1 and 2. The characteristic features of the Polyakov loops as an order parameter of the deconfinement transition are due to the Dirac string contributions alone. The photon part has a finite contribution for both phases and it changes only slightly. 
string tension (SU(2) MA)

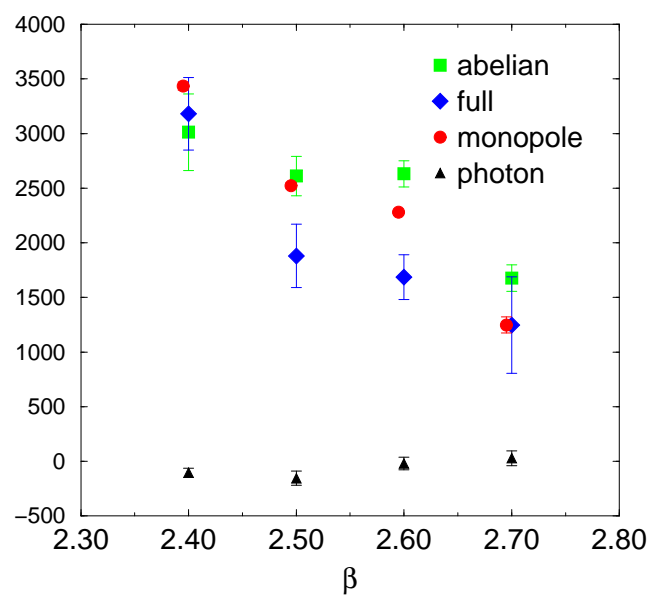

Fig.3 Monopole and photon contributions to the string tension in MA gauge in $S U(2)$ QCD in comparison with the full and the abelian ones.

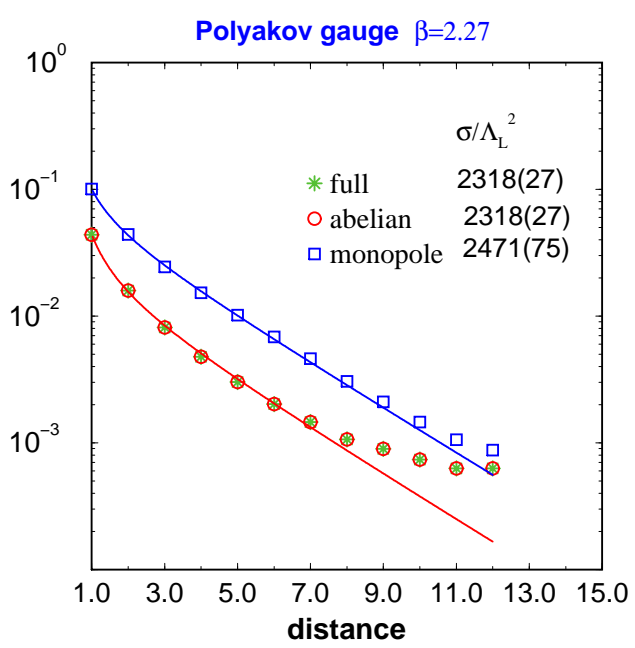

Fig.4 Polyakov loop correlations in the Polyakov gauge.

The fact that monopoles are responsible for the essential feature of the Polyakov loop is found also in $U(1)$ and in $S U(3)$. It is remarkable that the behavior of the abelian Polyakov loops as an order parameter and the monopole responsibility are seen in any gauge. 10 This is the first phenomena suggesting gauge independence of the 'tHooft conjecture.

\subsection{The string tension and monopoles}

Shiba and Suzukit . the full value of the string tension in $S U(2)$ QCD. An abelian Wilson loop operator is rewritten by a product of monopole and photon contributions. The string tensions evaluated from monopole and photon contributions are plotted in Fig.3. The string tension is reproduced only from the monopole contribution.

It is possible to know the static potential from the correlation of the Polyakov loops. The string tension is then derived from the static potential. We have measured the correlations using the Polyakov loop operators written in terms of full and abelian link fields and link fields given by the monopole Dirac string. The preliminary data are plotted in Fig.4 in the case of the Polyakov gauge. A linear behavior is seen with the almost similar string tension in all three cases. This is another data supporting gauge invariance of confinement mechanism due to monopole condensation. 


\section{Monopole action and condensation}

The above abelian dominance suggests that a set of $U(1)$ invariant operators $\{O(u(s, \mu))\}$ are enough to describe confinement. Then there must exists an effective $U(1)$ action $S_{\text {eff }}(u)$ describing confinement. We tried to derive $S_{\text {eff }}(u)$ using SchwingerDyson equations, but failed to get it in a compact and local form. $S_{\text {eff }}(u)$ contains larger and larger loops as $\beta$.

Shiba and Suzuki author tried to perform a dual transformation of $S_{\text {eff }}(u)$ in $S U(2)$ QCD and to obtain the effective $U(1)$ action A $11_{1}$ in terms of monopole currents.22 To study the long range behavior important in QCD, They have considered also extended monopoles.23 The extended monopole currents are defined by the number of the Dirac strings surrounding an extended cube:

$$
k_{\mu}^{(n)}(s)=\sum_{i, j, l=0}^{n-1} k_{\mu}(n s+(n-1) \hat{\mu}+i \hat{\nu}+j \hat{\rho}+l \hat{\sigma})
$$

where $k_{\mu}(s)$ is the ordinary monopole current.22 Considering extended monopoles corresponds to performing a block spin transformation on a dual latticet11 and so It is suitable for exploring the long range property of QCD.

The partition function of interacting monopole currents is expressed as

$$
Z=\left(\prod_{s, \mu} \sum_{k_{\mu}(s)=-\infty}^{\infty}\right)\left(\prod_{s} \delta_{\partial_{\mu}^{\prime} k_{\mu}(s), 0}\right) \exp (-S[k]) .
$$

It is natural to assume $S[k]=\sum_{i} f_{i} S_{i}[k]$. Here $f_{i}$ is a coupling constant of an interaction $S_{i}[k]$. For example, $f_{1}$ is the coupling of the self energy term $\sum_{n, \mu}\left(k_{\mu}(s)\right)^{2}$, $f_{2}$ is the coupling of a nearest-neighbor interaction term $\sum_{n, \mu} k_{\mu}(s) k_{\mu}(s+\hat{\mu})$ and $f_{3}$ is the coupling of another nearest-neighbor term $\sum_{n, \mu \neq \mu} k_{\mu}(s) k_{\mu}(s+\hat{\nu}) .7$ Shiba and Suzukit 6 extended a method developed by Swendsen 24 to the system of monopole currents obeying the current conservation rule.

The monopole actions are obtained locally enough for all extended monopoles considered even in the scaling region. They are lattice volume independent. The coupling constant $f_{1}$ of the self-energy term is dominant and the coupling constants decrease rapidly as the distance between the two monopole currents increases.

To study monopole dynamics, we have also studied the length of monopole loops and monopole charge distribution. We have found that 1)each vacuum has only one long connected loop and a small number of short loops. The difference of the two types of loops is very clear. 2)The length of the long loop becomes shorter as $\beta$ becomes larger. In the deep deconfinement region, there disappears a long loop. 3)Monopole charges are almost \pm 1 .

Since the long loop is important, we plot the value of monopole action versus length of the long loops as seen in Fig.5. The value of the action is proportional to the length $L$ of the long loop is well approximated by $f_{1} \times L$. 


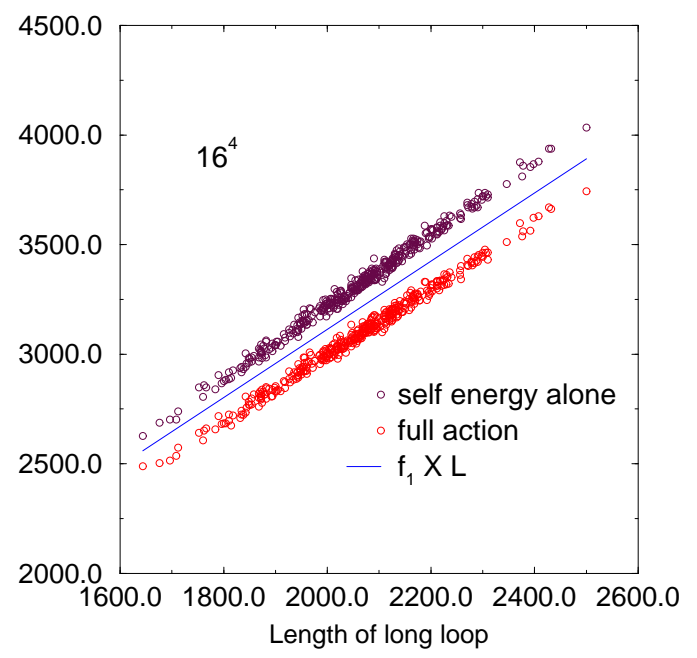

Fig.5 Monopole action versus length of loops

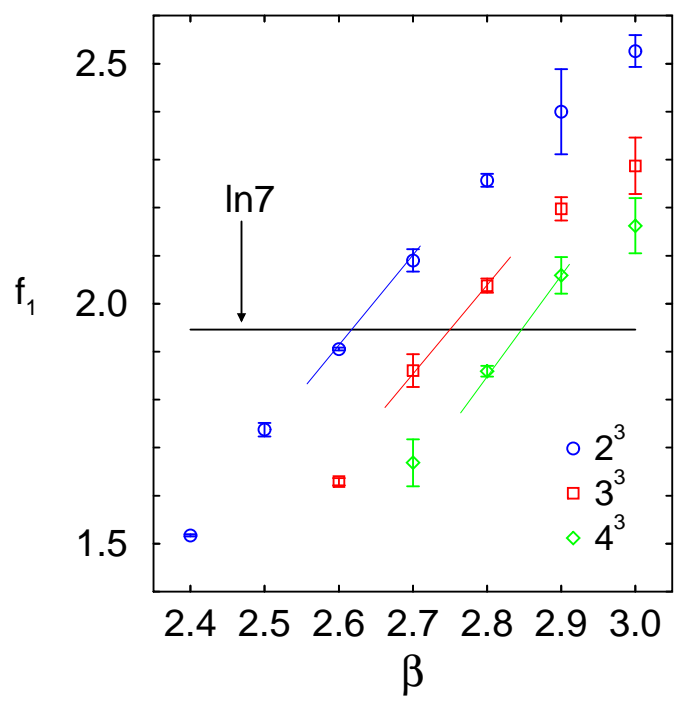

Fig. 6 Coupling constants $f_{1}$ versus $\beta$ for $2^{3}, 3^{3}$, and $4^{3}$ monopoles on $24^{4}$ lattice.

As done in compact QED, 25 the entropy of a monopole loop can be estimated as $\ln 7$ per unit loop length. Since the action is approximated by the self energy part $f_{1} L$, the free energy per unit monopole loop length is approximated by $\left(f_{1}-\ln 7\right)$ . If $f_{1}<\ln 7$, the entropy dominates over the energy, which means condensation of monopoles. In Fig.6, $f_{1}$ versus $\beta$ for various extended monopoles on $24^{4}$ lattice is shown in comparison with the entropy value $\ln 7$. Each extended monopole has its own $\beta$ region where the condition $f_{1}<\ln 7$ is satisfied. When the extendedness is bigger, larger $\beta$ is included in such a region. Larger extended monopoles are more important in determining the phase transition point.

The behaviors of $f_{i}$ are different for different extended monopoles. However, if we plot them versus $b=n \times a(\beta)$, we get a unique curve as in Fig.7. The coupling constants seem to depend only on $b$, not on the extendedness nor $\beta$. There is a critical $b_{c}$ corresponding to critical $\beta_{c}^{n}$, i.e., $b_{c}=n a\left(\beta_{c}^{n}\right)$.

Now we can derive important conclusions. Suppose the effective monopole action remains the same for any extended monopoles in the infinite volume limit. Then the finiteness of $b_{c}=n a\left(\beta_{c}^{n}\right)$ suggests $\beta_{c}^{n}$ becomes infinite when the extendedness $n$ goes to infinity. $S U(2)$ lattice QCD is always (for all $\beta$ ) in the monopole condensed and then in the color confinement phase 1 This is one of what one wants to prove in the 
Renormalization flow

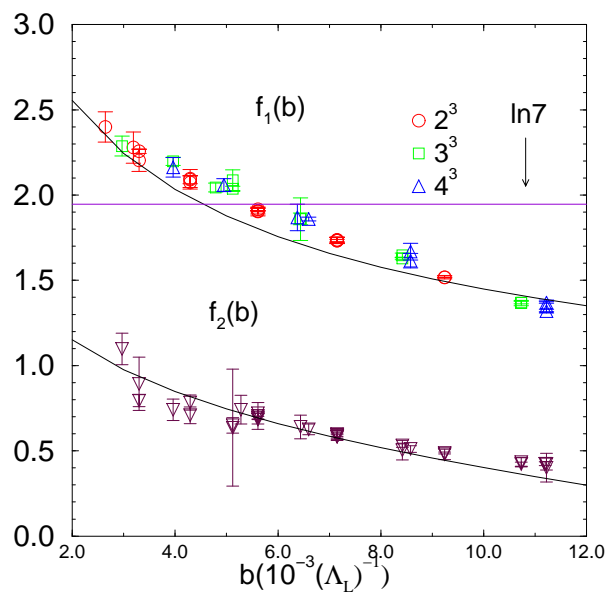

Fig.7 Coupling constants $f_{1}$ and $f_{2}$ versus $b$.

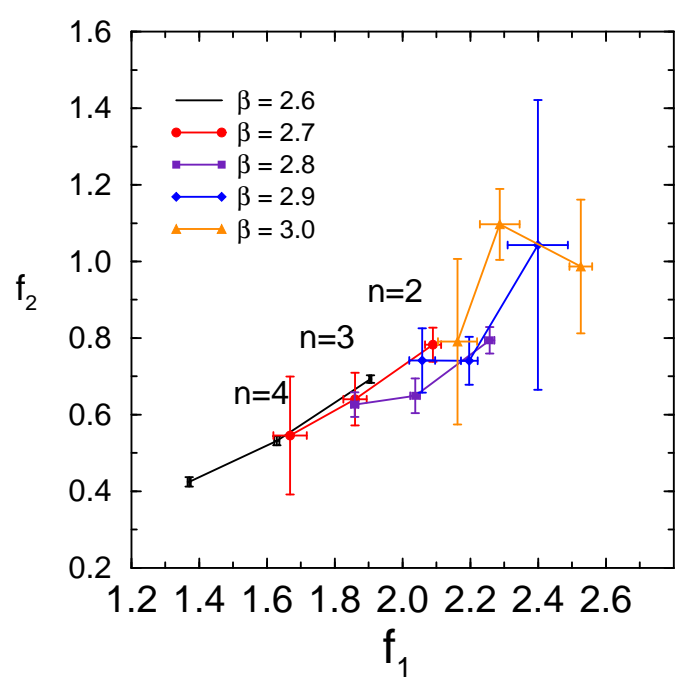

Fig.8 Renormalization flow in the $f_{1}-f_{2}$ plane

framework of lattice QCD.

Notice again that considering extended monopoles corresponds to performing a block spin transformation on the dual lattice. The above fact that the effective actions for all extended monopoles considered are the same for fixed $b$ means that the action may be the renormalized trajectory on which one can take the continuum limit. See Fig.8. Our results suggest the continuum monopole action takes the form predicted by Smit and Sijs.26

\section{Simulation of monopole action}

To test if the fixed monopole action is the renormalized trajectory, we have tried to simulate the system with the action. 5

We have obtained the following results:

1. The action with quadratic interactions alone is not good, since it gives a longer monopole loop and a larger string tension than expected. We need a term which gives a repulsive force between monopole currents.

2. With such small repulsive terms, we get the nice fit of the loop length and the string tension on small lattices. Finite-size effects are seen to be small.

3. We have also made Monte-Carlo simulations in the $T \neq 0$ QCD. Only the selfcoupling term can reproduce the qualitative features of the deconfinement transition as seen in Fig.10. 


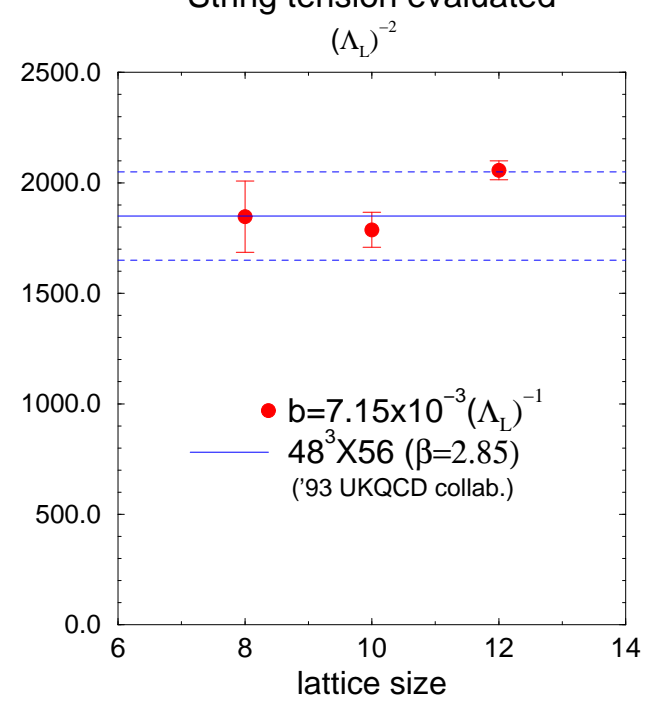

Fig.9 The string tension versus the lattice size

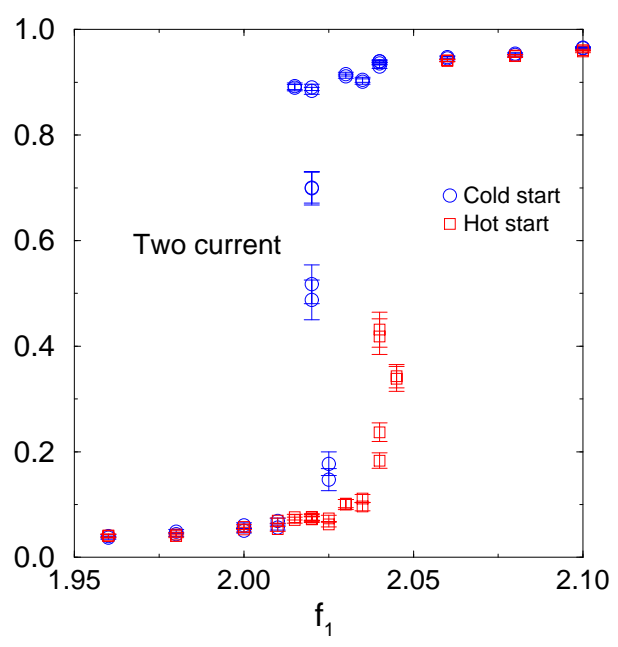

Fig.10 Polyakov loop from the self-coupling monopole interactions alone in the two-current case

\section{Discussions and outlook}

Some comments are in order.

1. It is important to find an order parameter of confinement due to monopole condensation. 15 The number of Dirac string $n_{i 4}(s)$ may be a candidate.15 $n_{i 4}(s)$ is a magnetic $U(1)$ variant quantity.27

2. Gauge independence should be proved if the monopole condensation is the real confinement mechanism. The data of Polyakov loops from monopoles are encouraging. That the correct string tension could be derived both in MA gauge and Polyakov gauge is also encouraging. Gauge independent results will be obtained if we go to large $\beta$ on larger lattice.

3. How to test the correctness of this idea? The theory predicts the existence of an axial vector glueball-like state $\mathrm{C}\left(J^{p c}=1^{+-}\right)$and a scalar glueball-like state $\chi\left(J^{p c}=0^{++}\right)$. The masses seem to satisfy $m_{c} \sim m_{\chi}$.28 30 The masses could not be too heavy. They have to exist under $2 \mathrm{Gev}$. To evaluate the correlation between the state and the light hadrons in MC simulations of full QCD is very important to derive the total width and the branching ratios. 
The author is thankful to Y.Matsubara, S.Kitahara, H.Shiba, S.Ejiri and O.Miyamura for (partial) collaboration and fruitful discussions. This work is financially supported by JSPS Grant-in Aid for Scientific Research (B) (No.06452028).

\section{References}

1. G. 'tHooft, Nucl. Phys. B190 (1981), 455.

2. T. Suzuki and I. Yotsuyanagi, Phys. Rev. D42 (1990), 4257; Nucl. Phys. B(Proc. Suppl.) 20 (1991), 236.

3. T. Suzuki, Nucl. Phys. B(Proc. Suppl.) 30 (1993), 176 and references therein.

4. H.Shiba and T.Suzuki, Nucl. Phys. B(Proc. Suppl.) 34 (1994), 182.

5. H.Shiba and T.Suzuki, Phys. Lett. B333 (1994), 461.

6. H.Shiba and T.Suzuki, Phys. Lett. B343 (1995), 315.

7. H.Shiba and T.Suzuki, To appear in Physics Letters B.

8. S.Ejiri, S.Kitahara, Y.Matsubara and T.Suzuki, Phys. Lett. B343 (1995), 304.

9. S.Ejiri, S.Kitahara, Y.Matsubara and T.Suzuki, Talk at Lattice 94, to appear in Nucl. Phys. B(Proc. Suppl.).

10. T. Suzuki et al., Phys. Lett. B347 (1995), 375.

11. T. Suzuki and H. Shiba, Talk at Lattice 94, to appear in Nucl. Phys. B(Proc. Suppl.).

12. Y.Matsubara et al., Talk at Lattice 94, to appear in Nucl. Phys. B(Proc. Suppl.).

13. S. Kitahara et al., Prog. Theor. Phys.93 (1995) 1.

14. T.Suzuki, Review talk at YITP workshop 'From Hadronic matter to Quark Matter' held at YITP, Kyoto University from Oct.30 till Nov.1 ,1994. Kanazawa Univ. Preprint KANAZAWA 94-26 (1994). To appear in Suppl. Prog.Theor.Phys..

15. T.Suzuki, Review talk at German-Japan Seminar 'QCD on Massively Parallel Computers' held at Yamagata from Mar.16 till 18 ,1995. Kanazawa Univ. Preprint KANAZAWA 95-02 (1995). To appear in Suppl. Prog.Theor.Phys..

16. T.Suzuki, Prog. Theo. Phys. 81 (1989), 752;

S.Maedan and T.Suzuki, Prog. Theo. Phys. 81 (1989), 229.

17. S.Maedan, Y.Matsubara and T.Suzuki Prog. Theo. Phys. 84 (1990), 130.

18. H.Monden et al., Phys. Lett. B294 (1992), 100.

19. S.Kamizawa et al., Nucl. Phys. B389 (1993), 563.

20. H. Kodama et al., in preparation.

21. A.S. Kronfeld et al., Phys. Lett. B198 (1987), 516.

A.S. Kronfeld et al., Nucl. Phys. B293 (1987), 461.

22. T.A. DeGrand and D. Toussaint, Phys. Rev. D22 (1980), 2478.

23. T.L. Ivanenko et al., Phys. Lett. B252 (1990), 631.

24. R.H. Swendsen,Phys. Rev. Lett. 52 (1984), 1165; Phys. Rev. B30 (1984), 3866 , 3875.

25. T.Banks et al., Nucl. Phys. B129 (1977), 493.

26. J. Smit and A.J. van der Sijs, Nucl. Phys. B355 (1991), 603.

27. A.Jevicki and P.Senjanovic, Phys. Rev.D11 (1975) 860.

28. V.Singh et al., LSU Preprint LSU HEP-1-92 (1992).

29. Matsubara et al., Nucl, Phys. B(Proc.Suppl.) 34 (1994) 176.

30. R.W.Haymaker, Nucl, Phys. B(Proc.Suppl.) 30 (1993) 568. 